OPEN ACCESS

Edited by:

Paul Comper,

University of Toronto, Canada

Reviewed by:

Pierre Langevin,

Laval University, Canada

Nicholas Baird Holowka,

University at Buffalo, United States

${ }^{*}$ Correspondence:

John J. Fraser

john.j.fraser8.mil@mail.mil orcid.org/0000-0001-9697-3795

Specialty section:

This article was submitted to Injury Prevention and Rehabilitation,

a section of the journal

Frontiers in Sports and Active Living

Received: 01 May 2021 Accepted: 05 July 2021 Published: 10 August 2021

Citation:

Fraser JJ, VanDehy J, Bodell DM, Gottshall KR and Sessoms PH (2021) Head and Body Dyskinesia During Gait in Tactical Athletes With Vestibular Deficit Following Concussion Front. Sports Act. Living 3:703982.

doi: 10.3389/fspor.2021.703982

\section{Head and Body Dyskinesia During Gait in Tactical Athletes With Vestibular Deficit Following Concussion}

\author{
John J. Fraser ${ }^{1 *}$, Jacob VanDehy ${ }^{1,2}$, Dawn M. Bodell ${ }^{1,2}$, Kim R. Gottshall ${ }^{2,3}$ and \\ Pinata H. Sessoms ${ }^{1}$
}

'Warfighter Performance Department, Operational Readiness and Health Directorate, Naval Health Research Center, San Diego, CA, United States, ${ }^{2}$ Leidos, Inc., San Diego, CA, United States, ${ }^{3}$ Florida Ear and Balance Center, Kissimmee, FL, United States

Background: Vestibular deficit is common following concussion and may affect gait. The purpose of this study was to investigate differences in head and pelvic center of mass (COM) movement during gait in military tactical athletes with and without concussion-related central vestibular impairment.

Material and Methods: 24 patients with post-concussion vestibular impairment (20 males, 4 females; age: $31.7 \pm 7.9$ years; BMl: $27.3 \pm 3.3$ ) and 24 matched controls (20 males, 4 females; age: $31.8 \pm 6.4$ years; BMl: $27.2 \pm 2.6)$ were included in the analyses. Three-dimensional head and pelvic displacement and velocities were collected at a 1.0 $\mathrm{m} / \mathrm{s}$ standardized treadmill walking speed and assessed using Statistical Parametric Mapping $t$-tests. Maximum differences $\left(d_{\max }\right)$ between groups were reported for all significant kinematic findings.

Results: The Vestibular group demonstrated significantly diminished anteroposterior head excursions $\left(d_{\max }=2.3 \mathrm{~cm}, p=0.02\right)$ and slower anteroposterior $\left(d_{\max }=0.37\right.$ $\mathrm{m} / \mathrm{s}, p=0.01)$, mediolateral $\left(d_{\max }=0.47 \mathrm{~m} / \mathrm{s}, p=0.02\right)$ and vertical $\left(d_{\max }=0.26 \mathrm{~m} / \mathrm{s}\right.$, $p<0.001)$ velocities during terminal stance into pre-swing phases compared to the Control group. Vertical pelvic excursion was significantly increased in midstance $\left(d_{\max }=\right.$ $2.4 \mathrm{~cm}, p=0.03$ ) and mediolaterally during pre- to initial-swing phases $\left(d_{\max }=7.5 \mathrm{~cm}\right.$, $p<0.001)$ in the Vestibular group. In addition, pelvic velocities of the Vestibular group were higher mediolaterally during midstance $\left(d_{\max }=0.19 \mathrm{~m} / \mathrm{s}, p=0.02\right)$ and vertically during post-initial contact $\left(d_{\max }=0.14 \mathrm{~m} / \mathrm{s}, p<0.001\right)$ and pre-swing $\left(d_{\max }=0.16 \mathrm{~m} / \mathrm{s}\right.$, $p<0.001)$ compared to the Control group.

Significance: The Vestibular group demonstrated a more constrained head movement strategy during gait compared with Controls, a finding that is likely attributed to a neurological impairment of visual-vestibular-somatosensory integration.

Keywords: movement, walking, dizziness, brain injuries, impairment 


\section{INTRODUCTION}

Concussion, also known as mild traumatic brain injury (mTBI), is a common clinical entity experienced by more than 15thousand military tactical athletes serving in the United States Armed Forces annually (DoD worldwide numbers for TBI, 2016) and is responsible for more than 2.8 million civilian emergency room visits per year (Taylor, 2017). Many individuals who sustain a concussion experience signs and symptoms that include dizziness, imbalance, dyskinesis, and cognitive deficit (Sosnoff et al., 2008). These manifestations are a result of neurophysiological impairment in higher-order brain function, pyramidal and extrapyramidal motor pathways, and vestibular function (Kolev and Sergeeva, 2016). These alterations in visualvestibular-somatosensory integration may persist well-beyond the initial injury. Athletes with prior history of concussion have been found to have lasting alteration in static and dynamic postural control at an average 44 months postinjury (Sosnoff et al., 2011). Visual-vestibular-somatosensory integration deficits may affect smooth pursuit, vestibulo-ocular reflex (VOR), dynamic visual acuity, gaze stabilization, subjective visual vertical, and spatial orientation during execution of complex motor tasks such as walking (Han et al., 2011). Due to the high demands required by military tactical athletes, (Scofield and Kardouni, 2015) these impairments can have substantial deleterious effects on physical function.

Individuals with concussion have been reported to have gait differences compared with healthy controls. In a recent systematic review assessing gait changes following concussion, individuals have been found to walk slower in the early acute phase (6 of 14 studies) that resolved within 10 days (10 of 13 studies) (Fino et al., 2018). There were mixed results for measures of stride length acutely (decreased in 2 of 7 studies) and double support time (increased in 2 of 5 studies) from intermediate to long-term time-points following injury (Fino et al., 2018). Decreased anteroposterior (4 of 13 studies) and increased mediolateral center of mass (COM) movement (3 of 12 studies) was observed acutely following injury that was primarily resolved by 10 days post-injury ( 8 of 9 studies) (Fino et al., 2018). It is highly likely that the wide variability of findings observed during gait is related to heterogeneity of neurological impairment found in this clinical population.

Little is known regarding the effects of central vestibular impairment on head and pelvic kinematics during walking following concussion. While prior studies have assessed head movement in individuals with peripheral vestibular impairment, (Pozzo et al., 1991; Mijovic et al., 2014) these findings may not be generalizable to individuals with central vestibular deficit. In a preliminary study of head and trunk mechanics during gait, military tactical athletes with vestibular deficit resulting from concussion were found to have asynchrony and large variability of the overall head position in relation to the pelvic COM (Sessoms et al., 2015). These findings warrant further investigation and replication to elucidate if the observed group variability and asynchrony is a function of movement variability or a small sample size. In addition, a more detailed analysis of the coordination between head and trunk movement could help establish potential causes for visual-vestibular impairments. Therefore, the purpose of this study was to investigate head and trunk mechanics during gait in military tactical athletes with persistent vestibular deficit following concussion compared to healthy controls.

\section{MATERIALS AND METHODS}

A descriptive laboratory cross-sectional study was performed where the independent variable was group (Control, Vestibular). The outcome measures were head and pelvis displacement and velocity in the sagittal, frontal, and transverse planes during treadmill walking at a standardized $1.0 \mathrm{~m} / \mathrm{s}$.

\section{Participants}

After excluding one participant for suspected secondary gain (i.e. malingering), 24 tactical athletes serving in the United States Armed Forces with post-concussion vestibular impairment (20 males, four females; age: $31.7 \pm 7.9$ years; BMI: $27.3 \pm 3.3$ ) recruited from a military vestibular rehabilitation clinic were included in the analyses. The control group was comprised of 24 military tactical athletes without a history of concussion (20 males, four females; age: $31.8 \pm 6.4$ years; BMI: 27.2 \pm 2.6) that were matched on sex, age, and BMI (Table 1). Participants in both groups were included if they were active duty military between the ages of 18 and 50. Healthy controls must have passed their most recent physical fitness assessment. Participants who incurred a concussion 6 to 52 weeks prior to consent and had a medical diagnosis of persistent concussionrelated central vestibular disorder were included in the postconcussion Vestibular group [Dizziness Handicap Inventory: $41.0 \pm 19.9$; Activities-specific Balance Confidence Scale: 73.7 \pm 17.1; Functional Gait Assessment: $23.8 \pm 4.5$; Computerized Dynamic Posturography Sensory Organization Test (SOT) Composite: $63.1 \pm 12.1$ ]. The differential diagnosis of persistent central vestibular deficit was made by an otorhinolaryngologist in consultation with an audiologist and physical therapist specializing in vestibular rehabilitation. More details regarding etiology, clinical differentiation, and diagnosis of this condition can be found in the work by Dougherty et al. (2020) and Reneker et al. (2018).

Participants were excluded from participation if they had an orthopedic condition that could affect gait, impaired joint mobility, benign positional vertigo, fluctuating peripheral vestibular dysfunction, non-organic behavior, conversion reactions, peripheral neuropathy, oculomotor nuclei (III, IV, VI) abnormalities, motor impairment, central neurological diseases, cerebral vascular accident, or were pregnant. Each participant gave informed consent before participating in the study. While participants did not directly contribute to the design of this study, specialist clinicians who care for and advocate for this patient population contributed. Regarding dissemination, study participants will be provided a link to a website that explains the research findings in common language. This study was approved 
TABLE 1 | Group demographics and spatiotemporal gait characteristics.

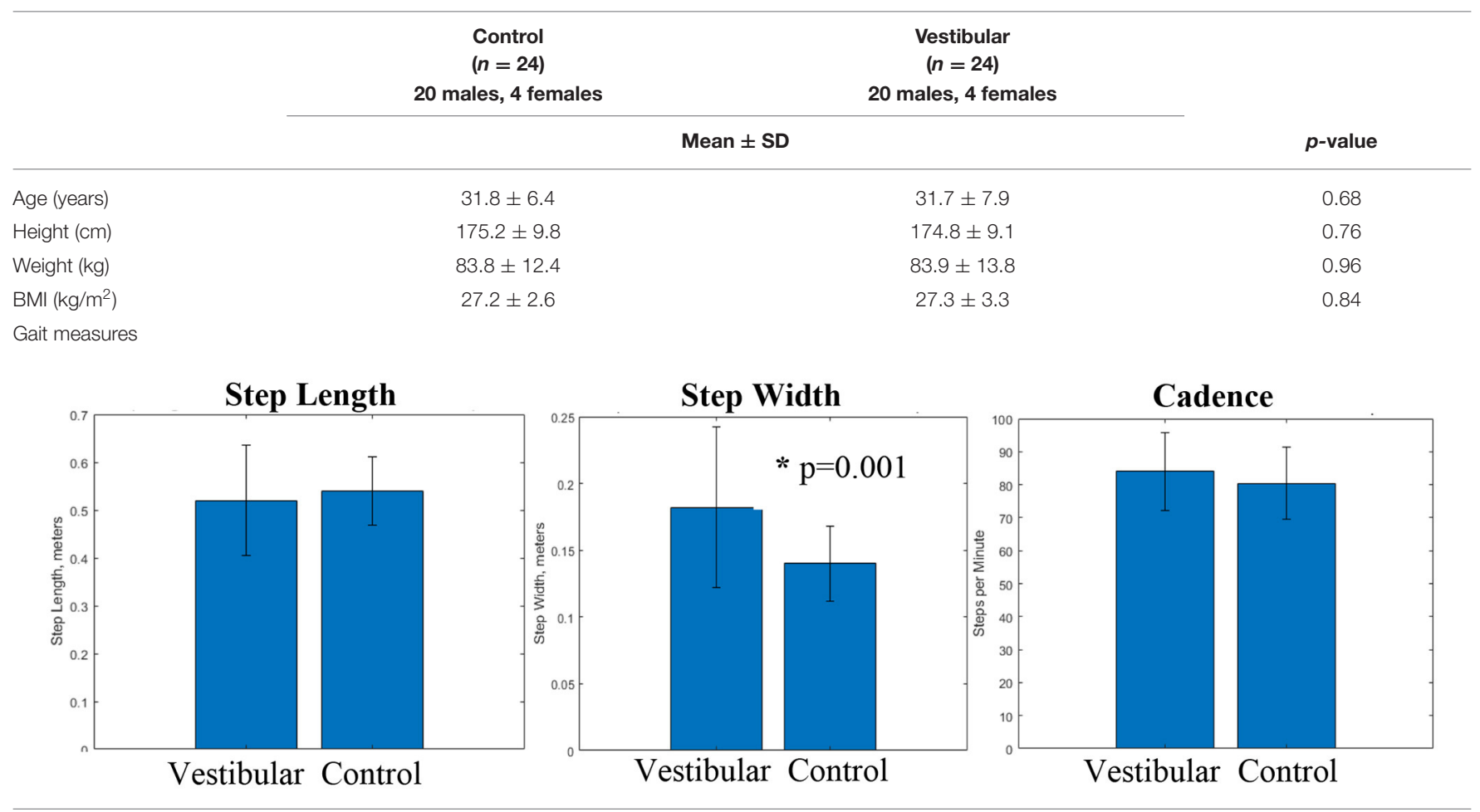

*statistically significant. BMI, body mass index; cm, centimeter; kg, kilogram; m, meter; min, minute; s, second.

by the Naval Health Research Center Institutional Review Board (protocol NHRC.2015.0010).

\section{Procedures}

Figure 1 details the study flow sheet from recruitment to analysis. Following consent, patients provided demographic information, and health and injury history. Height and weight were measured. Patients were asked to walk at a $1.0 \mathrm{~m} / \mathrm{s}$ pace on a dual-belt instrumented treadmill (Motekforce Link, Amsterdam, The Netherlands) while looking forward at an optotype placed on a screen in front of the treadmill. The use of a projected optotype during collection was employed to ensure the participant maintained their head and gaze forward during walking. Participants wore a safety harness to prevent them from falling but the harness did not provide weight support.

Kinematic data were collected with a 14-camera optical motion capture system (Motion Analysis Corp., Santa Rosa, CA, USA) at $120 \mathrm{~Hz}$ and referenced to the world. Head movement was tracked using a total of five photo-reflective markers affixed using adhesive to a fitted ball cap worn firmly on the head (left, front, right, back, and top of the head). A cluster of four markers affixed to a rigid plastic plate was positioned over the sacrum and fastened using a snug belt to measure pelvic motion. The movement of the centroid of the marker clusters was used as a surrogate for COM movement of the head and pelvis. A single heel marker affixed to the counter of each shoe was used to demarcate initial contact during gait. The Supplementary Figure 1 details the marker placement employed in this study. Three-dimensional measurements of head and pelvic displacement and velocity were obtained throughout the gait cycle during walking. Kinematic data of 10-15 consecutive strides were averaged using 101 data points and were processed using the Visual3D software (C-Motion Inc., Germantown, MD, USA). Head and pelvic COM were calculated as the average $\mathrm{X}, \mathrm{Y}, \mathrm{Z}$ position of the head and pelvic markers, respectively, while foot contact positions were collected using the heel markers. Since centroid positions were referenced to the world and likely migrated in the three-dimensional space during treadmill walking, kinematics were normalized to initial contact of each stride. Velocities were calculated as the first derivative of the positional data.

\section{Statistical Analysis}

An a priori sample size estimation of 16 participants were needed based on the variance of positional head movement,[6] an $\alpha$ $=0.05$, and $\beta=0.20$. Descriptive statistics were calculated for demographic and spatiotemporal gait characteristics and assessed for differences with independent $t$-tests using MATLAB (MathWorks, Inc., Natick, MA, USA). Group differences in triplanar motion of the head and pelvis over the gait cycle were assessed using Statistical Parametric Mapping (SPM) $t$ tests, a commonly used analytical approach for time-based analysis of biomechanical waveforms (Pataky et al., 2013). SPM1d version 0.4 was employed for analysis, a package written by Pataky (2012) for one-dimensional SPM analysis in Python 3.6.5 (Python Software Foundation, Beaverton, OR, 


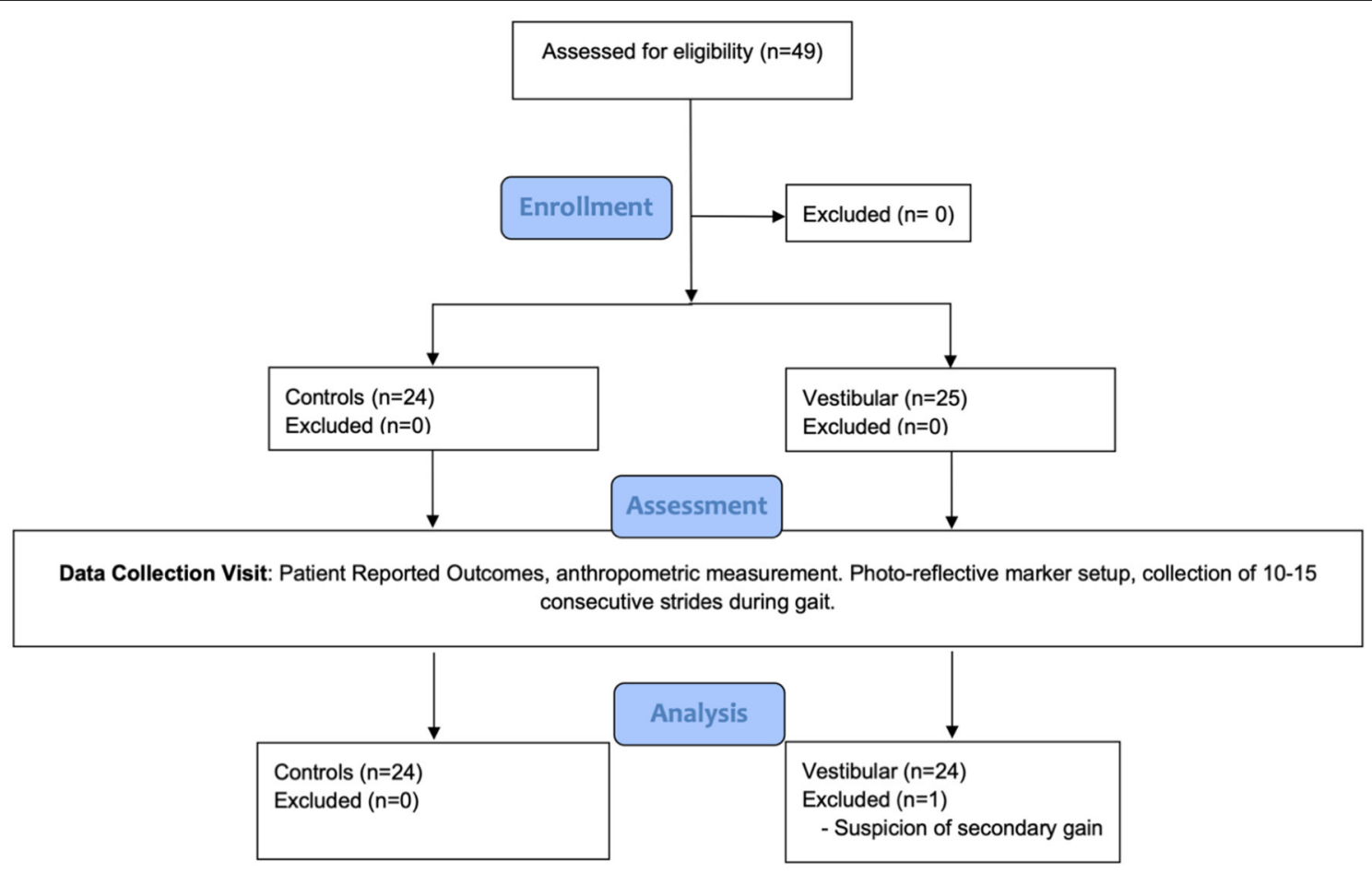

FIGURE 1 | Study flow diagram.

USA). Maximum difference $\left(\mathrm{d}_{\text {max }}\right)$ and mean difference $\left(\mathrm{d}_{\text {mean }}\right)$ between the two groups were reported for all significant kinematic findings.

\section{RESULTS}

There were no significant differences between the Vestibular and Control groups for age, height, mass, or BMI (Table 1). The Vestibular group walked with a significantly wider base of support (Table 1). There were no significant gait differences in step length or cadence between groups.

Figure 2 illustrates the COM motion of the head and pelvis in three dimensions during gait. Several significant group differences were observed in head and pelvic COM movement (excursion and velocity) during gait (Figure 3). The Vestibular group demonstrated diminished anteroposterior head excursions $\left(d_{\max }=2.3 \mathrm{~cm}, p=0.02\right)$ and slower anteroposterior $\left(d_{\max }=0.34 \mathrm{~m} / \mathrm{s}, p<0.001\right)$, mediolateral $\left(d_{\max }=0.47 \mathrm{~m} / \mathrm{s}, p=0.02\right)$ and vertical $\left(d_{\max }=0.26 \mathrm{~m} / \mathrm{s}\right.$, $p<0.001)$ velocities during terminal stance into pre-swing phases compared to controls. Vertical pelvic COM excursion was significantly increased in midstance $\left(d_{\max }=2.4 \mathrm{~cm}, p=0.03\right)$ and mediolaterally during pre- to initial-swing phases $\left(d_{\text {max }}=\right.$ $7.5 \mathrm{~cm}, p<0.001)$ in the Vestibular group. In addition, pelvic COM velocities were higher mediolaterally during midstance $\left(d_{\max }=0.19 \mathrm{~m} / \mathrm{s}, p=0.02\right)$ and vertically during post-IC $\left(d_{\max }=0.14 \mathrm{~m} / \mathrm{s}, p<0.001\right)$ and pre-swing $\left(d_{\max }=0.16\right.$ $\mathrm{m} / \mathrm{s}, p<0.001)$ in the Vestibular group compared to the Control group.

\section{DISCUSSION}

The primary findings of this study were that patients with postconcussion vestibular deficit demonstrated a more constrained head but increased mediolateral and vertical pelvic movement strategy during gait compared with the Control group, a finding that is likely attributed to a deficit of the VOR, vestibulospinal reflex, and dynamic balance integration. Alterations in pelvic motion during gait were likely a result of increased step width, a strategy employed to broaden the base of support and increase stability. Similarly, decreased head movement was likely compensatory to stabilize the eyes during gait. To our knowledge, these findings are the first to be reported within this clinical subpopulation.

\section{Head Mechanics}

We posit that the observed lower head excursion and velocity are likely a compensation for deficits in dynamic visual acuity (DVA), gaze stabilization, and subjective visual vertical affecting static and dynamic spatial orientation. From a kinematic perspective, movement of the head functions similarly to the proximal joints of the extremities. The mobility of the head allows for multiple degrees of freedom when exploring the environment visually. Similar to the extremities, proximal segmental stabilization of the head contributes to visual acuity, gaze stability, and spatial orientation. Vestibulopathy following concussion likely manifests with compensatory reduction of head excursion to try to stabilize gaze during dynamic gait. Further 


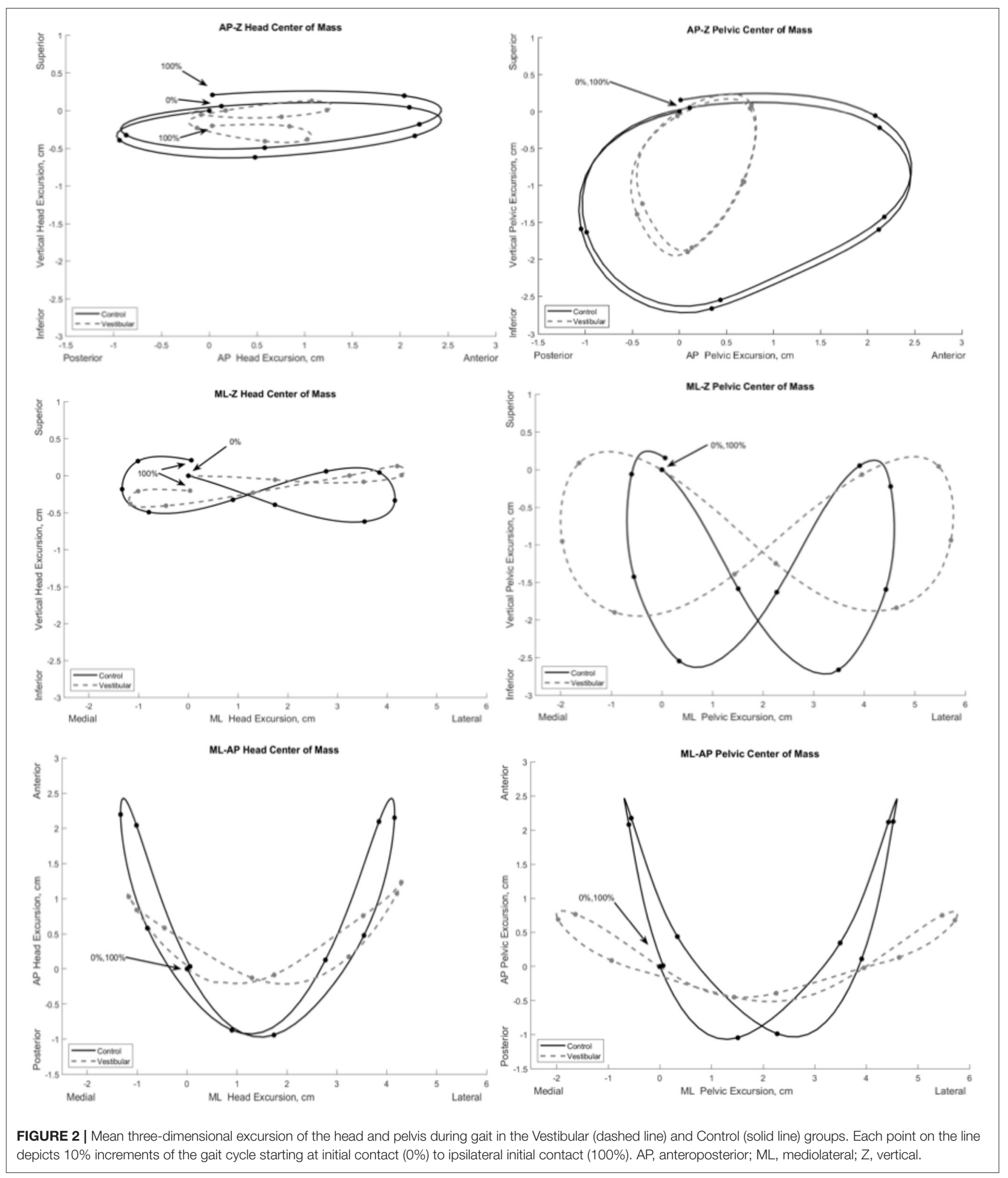

research incorporating optical tracking in relation to head movement during gait tasking is needed to substantiate this supposition.
The VOR, gaze stabilization, accurate subjective visual vertical, and the angular vestibulocollic (VCR) and cervicocollic (CCR) reflexes are important in head stabilization and control 

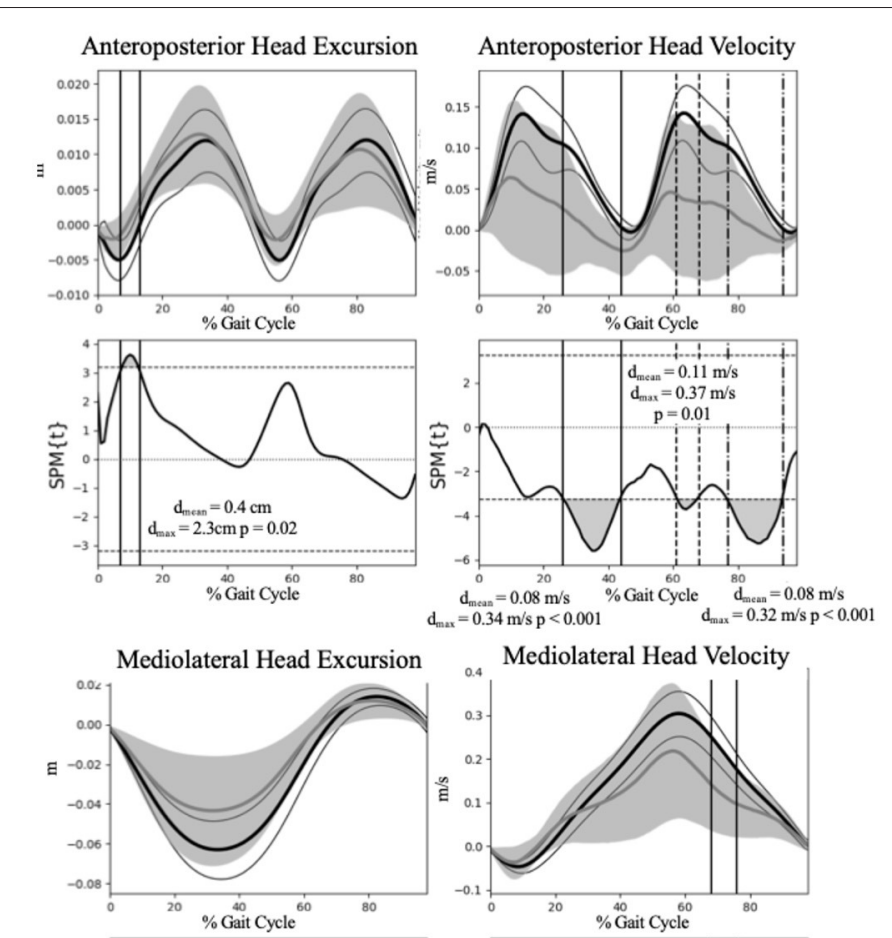

$d_{\max }=0.34 \mathrm{~m} / \mathrm{sp}<0.001$
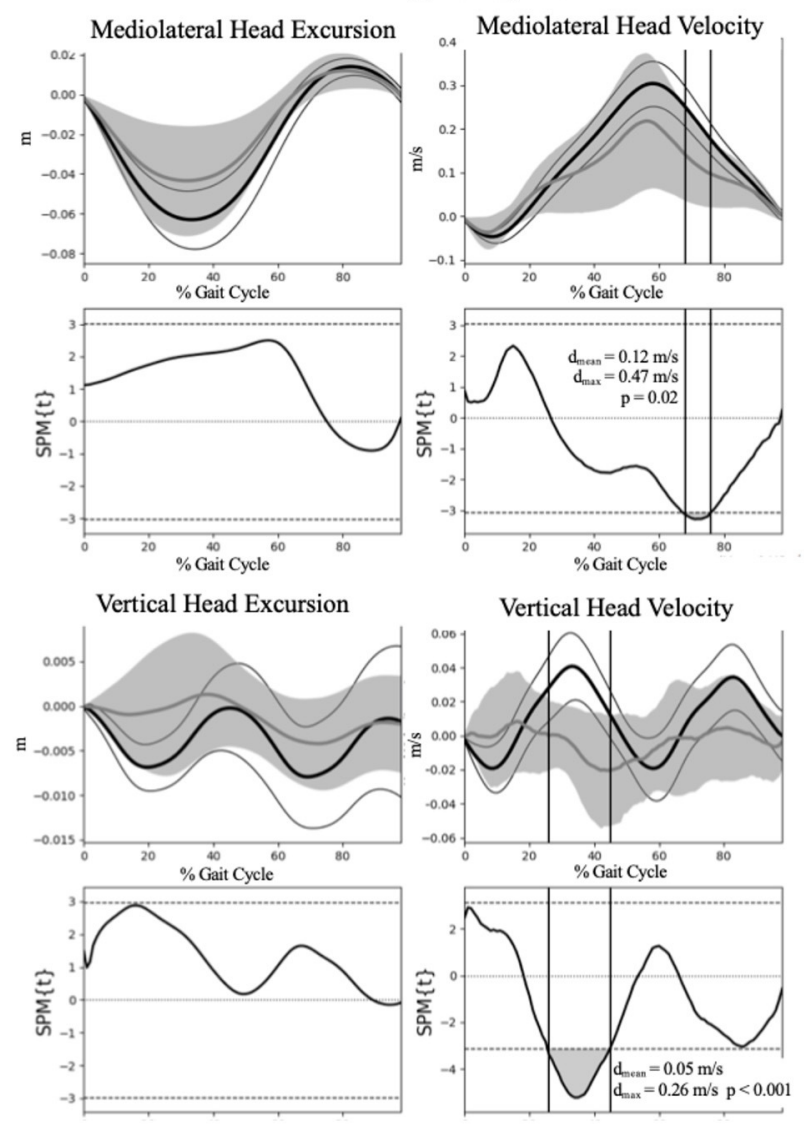
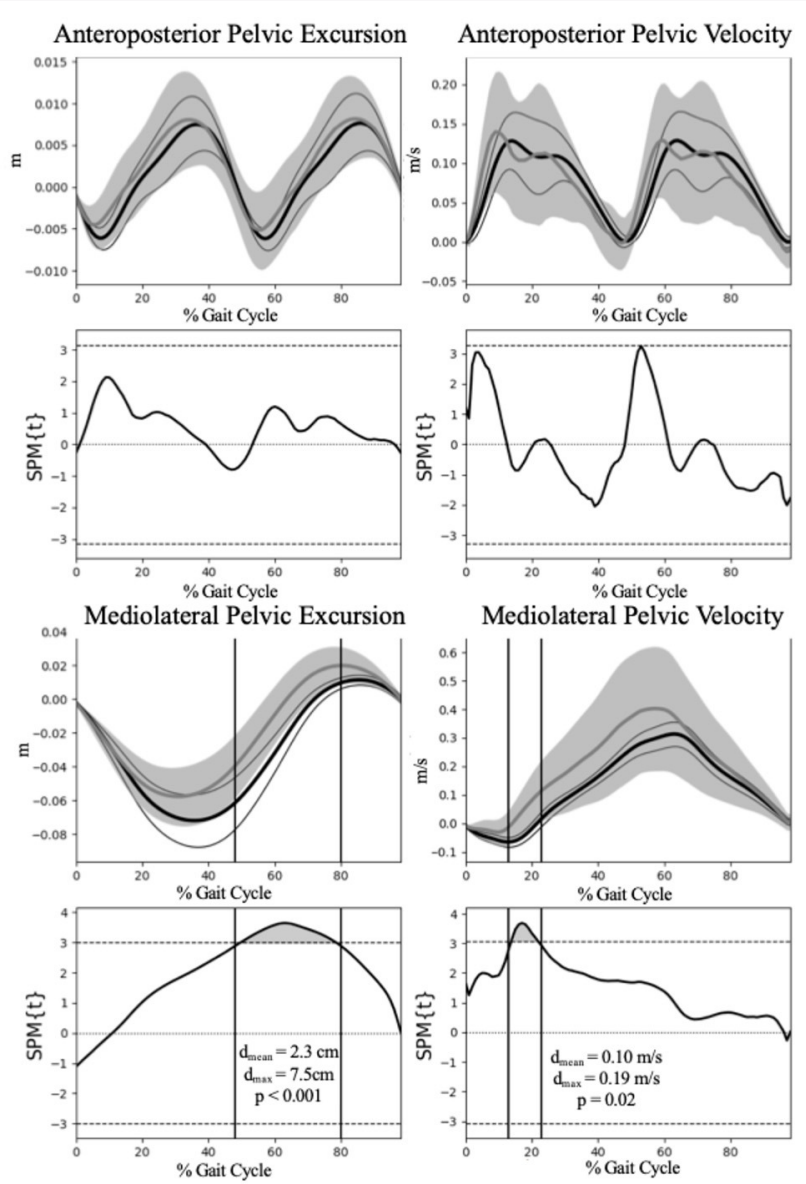

Vertical Pelvic Excursion
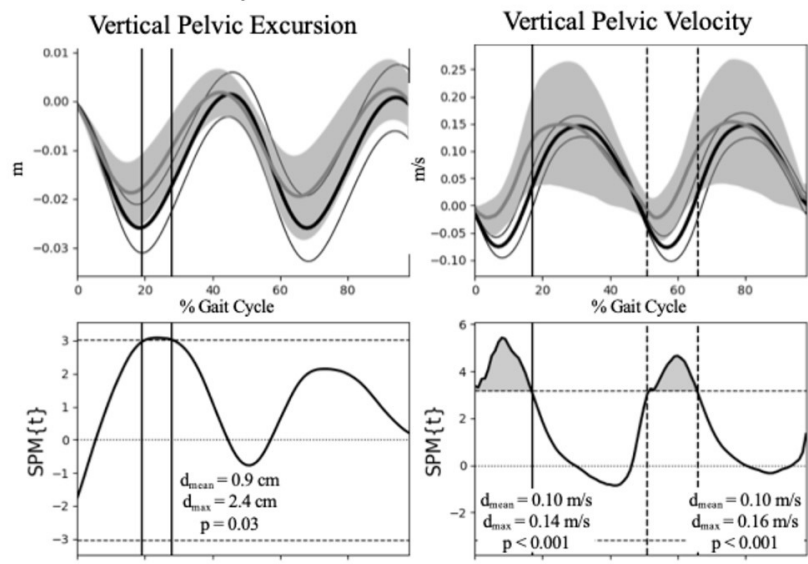

FIGURE 3 | Group comparison of the head and pelvis excursion and velocity during gait. Group means and 95\% confidence interval cloud for the Vestibular (gray mean line and confidence interval cloud) and Control (black mean line and white confidence interval cloud) groups are depicted. $d_{\text {max }}$, maximum difference between groups; $d_{\text {mean }}$, mean difference between groups; SPM, Statistical Parametric Mapping.

during static and dynamic function (Peng and Hain, 1999). Increasing temporal stimulation of the semicircular canals, otoliths sensitive to gravity, and the eyes contribute to "velocity storage" in the vestibular nuclei (Laurens and Angelaki, 2011). Individuals post-concussion may display high-gain VOR and a propensity for motion intolerance. Decreased excursion of head motion response to external moments is a countermeasure for the conflicting sensory afference when gait tasking is superimposed. Both the VCR and the CCR function to dampen head movement (Peng and Hain, 1999) and are normally inhibited during gait (Goldberg and Cullen, 2011). Our findings suggest that central vestibular impairment resulting from concussion may disinhibit these reflexes during walking, (Bisdorff et al., 1997; Peng and Hain, 1999) resulting in diminished head movement during gait. 
Mechanical or neurophysiological impairment resulting from zygapophyseal or muscular injury in the cervical spine may also plausibly contribute to our finding of constrained head movement. Kinematics of concussion involved high accelerations of the head that are mitigated by cervical co-contraction (Eckner et al., 2014). Cervical spine pain is a common comorbidity following concussion, a condition that has many overlapping signs and symptoms (Morin et al., 2016). It is plausible that injury to the cervical joints and muscles that serve an important role in providing afferent information regarding head position is disrupted. Furthermore, injury may change the contraction dynamics of the cervical musculature (Peng and Hain, 1999) and further increase the burden on impaired central control mechanisms. Psychogenic factors are also a plausible contributor to diminished head movement excursion and velocity following concussion. Individuals with psychological trauma resulting from the injurious event or other prior traumatic exposure may demonstrate altered movement strategies. Kinesiophobia, pain, and perceived function have previously been shown to be associated with altered cervical kinematics (Sarig Bahat et al., 2014). These suppositions will need to be elucidated and should be considered as potential covariates in future research.

To contextualize our results, our data both agree and diverge from previous studies of head movement in patients with post-concussion central vestibular deficit and other studies investigating peripheral vestibular dysfunction. Sessoms et al. (2015) assessed head kinematics during walking in military tactical athletes with post-concussion vestibular deficit and found asynchrony and large group variability of head position in relation to the pelvic COM. We similarly observed substantially large group variability, a finding likely resulting from heterogeneity of movement strategies employed during walking in the Vestibular group. Mijovic et al. (2014) studied head movement in individuals with unilateral peripheral vestibular deficit during a 15 -meter walking task using movement sensors and found no kinematic differences compared to healthy controls. This is likely a function of severity of impairment, with our sample having substantially higher DHI scores compared to those reported by Mijovic et al. (2014). While Pozzo et al. (1991) found that individuals with bilateral peripheral vestibular deficit demonstrated no significant differences in head motion during walking measured with video analysis, they similarly observed greater variability in movement. Disparity between our findings and those previously reported are likely a function of lesion type and severity of symptoms. The review article by Karatas (2008) provides an excellent contextualization and contrast between central and peripheral vestibular deficit.

\section{Body Mechanics}

Basford et al. (2003) studied body COM kinematics in individuals with traumatic brain injury with similar DHI scores and found that body COM excursion and velocity were significantly diminished in the AP and increased in the ML compared to healthy controls. These findings were attributed to decreased walking velocity and step length (Basford et al., 2003), spatiotemporal alterations previously observed in this clinical population (Basford et al., 2003; McFadyen et al., 2003). The supposition of pelvic excursion as a function of spatiotemporal parameters is supported by our findings. While we similarly saw increased mediolateral and vertical pelvic motion and velocity, we did not observe any differences in anteroposterior parameters. Since gait speed was standardized, the increased mediolateral pelvic excursion was likely attributed to the wider step width, a finding likely attributed to the neurological deficit and an attempt to widen the base of support during this dynamic activity. This finding has similarly been observed in individuals with vestibulopathy during walking at a standardized velocity (Krebs et al., 2002).

The pelvic excursions and velocities observed in our study agree with a few previously-reported findings. Catena et al. (2007) studied pelvic COM kinematics during over-ground walking in individuals post-concussion and found that pelvic COM velocity were significantly increased in the ML, with significantly lower COM velocity in the AP. They did not find any significant differences in pelvic excursion (Catena et al., 2007). In a later study, Catena et al. (2009) found no significant differences in COM kinematics between post-concussion patients and healthy controls during single-task walking up to 28 days post injury. Parker et al. (2006) found that while AP displacement and velocity were not significantly different between post-concussion patients and healthy controls up to 28 days following concussion, there was a significantly diminished maximum separation difference between AP COM and center of pressure indicating a more conservative strategy for stability. Reciprocal arm swing has been postulated to be a mass dampener that mitigates trunk torsion and head yaw during walking (Pontzer et al., 2009). While we did not measure upper extremity kinematics, it is plausible that asynchronous arm swing contributed to alteration in pelvic and head mechanics in this study. This supposition should be evaluated in future study.

\section{Clinical and Research Implications}

Clinical and instrumented measures of head motion during gait may garner additional information regarding central neurological mechanisms and may be an important clinical correlate in the assessment of the balance system during function. Based on heterogeneity of clinical presentation following concussion, diminished head motion may be a useful measure when diagnosing, prognosticating, and guiding treatment in this population. Physical examination should include assessment of cervical motion, cervicogenic dizziness, dynamic visual acuity, gaze stabilization, subjective visual vertical, static balance using the SOT, and gait. Identified deficits to include diminished head kinematics during gait should be addressed during rehabilitation to allow the patient to reach their full potential. Further research is needed to investigate how eye tracking and stabilization is related to relative head motion during gait. Furthermore, it will also be interesting to assess how these factors change in response to vestibular rehabilitation. Lastly, we opted to have the participants focus on an optotype to maintain gaze forward. Future research is needed to elucidate how the 
factors of visual focus may influence head movement in this clinical population.

\section{Limitations}

There are limitations to this study. This was a cross-sectional study, so cause and effect relations cannot be determined. The study sample consisted of young adult military tactical athletes with a high level of physical function prior to injury, hence generalizability is likely limited to individuals of similar age and physical function. While there is always a possibility that the participants may have been misdiagnosed as having central vestibular deficit, we are confident in the diagnostic accuracy based on the findings of the extensive workup performed by the interdisciplinary team of specialists. Kinematic measurements were focused on assessment of the pelvic and head COM during walking. Three-dimensional kinematic evaluation of head, arm, trunk, and lower extremity kinematics were not possible based on the marker set used in this study. Future study should consider the intersegmental coupling relationships that can further elucidate neurophysiological constraint and variability in the kinematic chain during walking.

\section{CONCLUSION}

Patients with post-concussion vestibular deficit demonstrated a more constrained head and an altered pelvic movement strategy during gait compared with the Control group, a finding that is likely attributed to a neurological impairment of visual-vestibular-somatosensory integration. Clinicians and researchers should consider assessment of head and neck movement during gait following concussion, as this information may provide greater insight to impaired peripheral and central vestibular-visual-somatosensory mechanisms.

\section{DATA AVAILABILITY STATEMENT}

The datasets presented in this article are not readily available because participants of this study did not agree for their data to be shared publicly, so supporting data is not available. Requests to access the datasets should be directed to Pinata H. Sessoms, pinata.h.sessoms.civ@mail.mil.

\section{REFERENCES}

Basford, J. R., Chou, L.-S., Kaufman, K. R., Brey, R. H., Walker, A., Malec, J. F., et al. (2003). An assessment of gait and balance deficits after traumatic brain injury. Arch. Phys. Med. Rehabil. 84, 343-349. doi: 10.1053/apmr.2003.50034

Bisdorff, A. R., Bronstein, A. M., Wolsley, C., and Lees, A. J. (1997). Torticollis due to disinhibition of the vestibulo-collic reflex in a patient with Steele-Richardson-Olszewski syndrome. Mov. Disord. 12, 328-336. doi: $10.1002 /$ mds.870120311

Catena, R. D., van Donkelaar, P., and Chou, L.-S. (2007). Cognitive task effects on gait stability following concussion. Exp. Brain Res. 176, 23-31. doi: 10.1007/s00221-006-0596-2

\section{ETHICS STATEMENT}

The studies involving human participants were reviewed and approved by the Naval Health Research Center Institutional Review Board in compliance with all applicable Federal regulations governing the protection of human subjects. Research data were derived from an approved Naval Health Research Center Institutional Review Board protocol, number NHRC.2015.0010. All participants provided informed consent. The patients/participants provided their written informed consent to participate in this study.

\section{AUTHOR'S NOTE}

Presented at the Military Health System Research Symposium, August 20, 2018, Orlando, FL and is archived at doi: 10.7490/f1000research.1115992. The preprint of the manuscript is archived on medRxiv and accessible at doi: 10.1101/2021.04.27.21256200.

\section{AUTHOR CONTRIBUTIONS}

JF contributed to study design, data analysis and interpretation, and drafting this manuscript. JV contributed to data collection and analysis. DB contributed to study design, participant recruitment, data collection, and interpretation of findings. KG contributed to study design, data collection and interpretation, and drafting this manuscript. PS contributed to study design, procurement of funding, data collection and interpretation, and drafting this manuscript. All authors have reviewed and approved this manuscript.

\section{FUNDING}

This work was supported by the U.S. Navy Bureau of Medicine and Surgery under work unit no. N1703.

\section{SUPPLEMENTARY MATERIAL}

The Supplementary Material for this article can be found online at: https://www.frontiersin.org/articles/10.3389/fspor. 2021.703982/full\#supplementary-material

Supplementary Figure 1 | Photo-reflective marker placement for three-dimensional motion capture of the head and pelvis during walking.

Catena, R. D., van Donkelaar, P., and Chou, L.-S. (2009). Different gait tasks distinguish immediate vs. long-term effects of concussion on balance control. J. NeuroEngineering Rehabil. 6:25. doi: 10.1186/1743-0003-6-25

DoD worldwide numbers for TBI (2016). DVBIC. Available at: http://dvbic.dcoe. mil/dod-worldwide-numbers-tbi [accessed December 12, 2017]

Dougherty, J. M., Carney, M., and Emmady, P. D. (2020). "Vestibular Dysfunction," in StatPearls (Treasure Island (FL): StatPearls Publishing). Available online at: http://www.ncbi.nlm.nih.gov/books/NBK558926/ [Accessed June 16, 2021]

Eckner, J. T., Oh, Y. K., Joshi, M. S., Richardson, J. K., and AshtonMiller, J. A. (2014). Effect of neck muscle strength and anticipatory cervical muscle activation on the kinematic response of the head to 
impulsive load. Am. J. Sports Med. 42, 566-576. doi: 10.1177/03635465135 17869

Fino, P. C., Parrington, L., Pitt, W., Martini, D. N., Chesnutt, J. C., Chou, L.-S., et al. (2018). Detecting gait abnormalities after concussion or mild traumatic brain injury: a systematic review of single-task, dual-task, and complex gait. Gait Posture 62, 157-166. doi: 10.1016/j.gaitpost.2018.03.021

Goldberg, J. M., and Cullen, K. E. (2011). Vestibular control of the head: possible functions of the vestibulocollic reflex. Exp. Brain Res. 210, 331-345. doi: 10.1007/s00221-011-2611-5

Han, B. I., Song, H. S., and Kim, J. S. (2011). Vestibular rehabilitation therapy: review of indications, mechanisms, and key exercises. J. Clin. Neurol. Seoul. Korea. 7, 184-196. doi: 10.3988/jcn.2011.7.4.184

Karatas, M. (2008). Central vertigo and dizziness: epidemiology, differential diagnosis, and common causes. Neurologist 14, 355-364. doi: 10.1097/NRL.0b013e31817533a3

Kolev, O. I., and Sergeeva, M. (2016). Vestibular disorders following different types of head and neck trauma. Funct. Neurol. 31, 75-80. doi: 10.11138/FNeur/2016.31.2.075

Krebs, D. E., Goldvasser, D., Lockert, J. D., Portney, L. G., and Gill-Body, K. M. (2002). Is base of support greater in unsteady gait? Phys. Ther. 82, 138-147. doi: $10.1093 / \mathrm{ptj} / 82.2 .138$

Laurens, J., and Angelaki, D. E. (2011). The functional significance of velocity storage and its dependence on gravity. Exp. Brain Res. Exp. Hirnforsch Exp. Cerebrale 210, 407-422. doi: 10.1007/s00221-011-2568-4

McFadyen, B. J., Swaine, B., Dumas, D., and Durand, A. (2003). Residual effects of a traumatic brain injury on locomotor capacity: a first study of spatiotemporal patterns during unobstructed and obstructed walking. J. Head Trauma Rehabil. 18, 512-525. doi: 10.1097/00001199-200311000-00005

Mijovic, T., Carriot, J., Zeitouni, A., and Cullen, K. E. (2014). Head movements in patients with vestibular lesion: a novel approach to functional assessment in daily life setting. Otol. Neurotol. Off. Publ. Am. Otol. Soc. Am. Neurotol. Soc. Eur. Acad. Otol. Neurotol. 35, e348-e357. doi: 10.1097/MAO.00000000000 00608

Morin, M., Langevin, P., and Fait, P. (2016). Cervical spine involvement in mild traumatic brain injury: a review. J. Sports Med. 2016:1590161. doi: 10.1155/2016/1590161

Parker, T. M., Osternig, L. R., Van Donkelaar, P., and Chou, L.-S. (2006). Gait Stability following concussion. Med. Sci. Sports Exerc. 38, 1032-1040. doi: 10.1249/01.mss.0000222828.56982.a4

Pataky, T. C. (2012). One-dimensional statistical parametric mapping in Python. Comput. Methods Biomech. Biomed. Eng. 15, 295-301. doi: 10.1080/10255842.2010.527837

Pataky, T. C., Robinson, M. A., and Vanrenterghem, J. (2013). Vector field statistical analysis of kinematic and force trajectories. J. Biomech. 46, 2394-2401. doi: 10.1016/j.jbiomech.2013.07.031

Peng, G. C. Y., and Hain, T. C. (1999). Predicting vestibular, proprioceptive, and biomechanical control strategies in normal and pathological head movements. IEEE Trans. Biomed. Eng. 46, 1269-1280. doi: 10.1109/10. 797986

Pontzer, H., Holloway, J. H., Raichlen, D. A., and Lieberman, D. E. (2009). Control and function of arm swing in human walking and running. J. Exp. Biol. 212, 523-534. doi: 10.1242/jeb.024927

Pozzo, T., Berthoz, A., Lefort, L., and Vitte, E. (1991). Head stabilization during various locomotor tasks in humans. II. Patients with bilateral peripheral vestibular deficits. Exp. Brain Res. 85, 208-217. doi: 10.1007/BF002 30002

Reneker, J. C., Cheruvu, V. K., Yang, J., James, M. A., and Cook, C. E. (2018). Physical examination of dizziness in athletes after a concussion: a descriptive study. Musculoskelet Sci. Pract. 34, 8-13. doi: 10.1016/j.msksp.2017. 11.012
Sarig Bahat, H., Weiss, P. L., Sprecher, E., Krasovsky, A., and Laufer, Y. (2014). Do neck kinematics correlate with pain intensity, neck disability or with fear of motion? Man. Ther. 19, 252-258. doi: 10.1016/j.math.2013.10.006

Scofield, D. E., and Kardouni, J. R. (2015). the tactical athlete: a product of 21 st century strength and conditioning. Strength Cond. J. 37, 2-7. doi: 10.1519/SSC.0000000000000149

Sessoms, P. H., Gottshall, K. R., Sturdy, J., and Viirre, E. (2015). Head stabilization measurements as a potential evaluation tool for comparison of persons with TBI and vestibular dysfunction with healthy controls. Mil. Med. 180, 135-142. doi: 10.7205/MILMED-D-14-00386

Sosnoff, J. J., Broglio, S. P., and Ferrara, M. S. (2008). Cognitive and motor function are associated following mild traumatic brain injury. Exp. Brain Res. 187, 563-571. doi: 10.1007/s00221-008-1324-x

Sosnoff, J. J., Broglio, S. P., Shin, S., and Ferrara, M. S. (2011). Previous mild traumatic brain injury and postural-control dynamics. J. Athl. Train 46, 85-91. doi: 10.4085/1062-6050-46.1.85

Taylor, C. A. (2017). Traumatic brain injury-related emergency department visits, hospitalizations, and deaths-United States, 2007 and 2013. MMWR Surveill. Summ. 66, 1-16. doi: 10.15585/mmwr.ss6609a1

Author Disclaimer: The authors are military service members or employees of the U.S. Government and this work was prepared as part of their official duties. Title 17, U.S.C. $\$ 105$ provides that copyright protection under this title is not available for any work of the U.S. Government. Title 17, U.S.C. $\$ 101$ defines a U.S. Government work as work prepared by a military service member or employee of the U.S. Government as part of that person's official duties. Report No. 1969 was supported by the U.S. Navy Bureau of Medicine and Surgery under work unit no. N1703. The views expressed in this article are those of the authors and do not necessarily reflect the official policy or position of the Department of the Navy, Department of Defense, nor the U.S. Government. The study protocol was approved by the Naval Health Research Center Institutional Review Board in compliance with all applicable Federal regulations governing the protection of human subjects. Research data were derived from an approved Naval Health Research Center Institutional Review Board protocol, number NHRC.2015.0010. All participants provided informed consent.

Conflict of Interest: Authors JV, DB, and KG was employed by the company Leidos, Inc.

The remaining authors declare that the research was conducted in the absence of any commercial or financial relationships that could be construed as a potential conflict of interests.

Publisher's Note: All claims expressed in this article are solely those of the authors and do not necessarily represent those of their affiliated organizations, or those of the publisher, the editors and the reviewers. Any product that may be evaluated in this article, or claim that may be made by its manufacturer, is not guaranteed or endorsed by the publisher.

Copyright (C) 2021 This work was authored by John J. Fraser, Jacob Vandehy, Dawn M. Bodell, Kim R. Gottshall, and Pinata H Sessoms on behalf of the U.S. Government and, as regards Dr. Fraser, Dr. Vandehy, Dr. Bodell, Dr. Gottshall, Dr. Sessoms, and the U.S. Government, is not subject to copyright protection in the United States. Foreign and other copyrights may apply. This is an open-access article distributed under the terms of the Creative Commons Attribution License (CC BY). The use, distribution or reproduction in other forums is permitted, provided the original author(s) and the copyright owner(s) are credited and that the original publication in this journal is cited, in accordance with accepted academic practice. No use, distribution or reproduction is permitted which does not comply with these terms. 